\title{
Two-way interactive refinement of segmented medical volumes
}

\author{
Alberto Signoroni and Riccardo Leonardi \\ University of Brescia \\ Electronics for Automation Department, Telecommunications Group \\ via Branze, 38, I25123 Brescia, Italy \\ forename.surname@ing.unibs.it
}

\begin{abstract}
For complex medical image segmentation tasks which also require high accuracy, prior information must usually be generated in order to initialize and confine the action of the computational tools. This can be obtained by task oriented specialization layers operating within automatic segmentation techniques or by advanced exploitation of userdata interaction, in this case the segmentation technique can conserve generality and results can be inherently validated by the user itself, in the measure he is allowed to effectively steer the process towards the desired result. In this paper we present a highly accurate and still general morphological $3 D$ segmentation system where rapid convergence to the desired result is guaranteed by a two-way interactive segmentation-refinement loop, where the flow of prior information is inverted (from computing tools to the user) in the refinement phase in order to help the user to quickly select most effective refinement strategies.
\end{abstract}

\section{Introduction}

Accuracy and generality are usually conflicting aspects in segmentation systems, especially when complex and critical tasks are involved, as it is the case of the segmentation of biomedical volumes for clinical applications. In this field, accuracy mainly concerns the possibility to generate satisfactory results to be used in subsequent analysis steps such as visualization or quantification of the desired anatomical or pathological structures. Due to the exploitation of a mixed high-level (from semantics) and low-level (from data features) information which typically constitute an ineluctable condition for an accurate segmentation, successful segmentation methods for biomedical images are typically of two kinds: a) highly task specialized automatic methods, or b) user-steered or interactive methods (see [4] for a useful review). The second kind of methods are also usually conceived to be specialized with respect to a task to be accomplished, and then they may suffer from the presence of alternative automatic solutions [3]. Therefore, an interesting field of investigation is the one which studies interactive systems and synergic computational technologies that are able to guarantee, at the same time, a high level of accuracy and a sufficient degree of generality. Generality can be defined as the capability to deal with all problems of a certain, suitably wide, class (characterized by a certain amount of a-priori knowledge about the segmentation problem nature) despite the inherent degree of unpredictability (e.g. due to anatomic and pathological variety) and the discrimination difficulties (e.g. due to the physical signal and noise limitations on the image information content). Generality is a valuable feature because techniques that are conceived to deal with general tasks are usually well adapted to be specialized for dedicated tasks (specialization can be modeled in terms of additional a-priori knowledge). The opposite is, in general, not true. Moreover, generality manifests its great value when the system should face with the unpredictable aspects of pathological situations: in fact, many automatic as well as guided segmentation systems do a fine job on normal data-sets while they may perform "unpredictably" in pathological situations.

Generality may have the apparent cost of admitting segmentation procedures which do not always guarantee successful results in one stroke. In other terms, some kind of refinement must taken into account and one major problem in such systems is to guarantee a rapid convergence to the desired result. In particular, for interactive segmentation systems, such a convergence is critically related to the cooperation and consistence between the user data-interaction language and the computational engines. Unfortunately, while in a user perspective the task of guiding a segmentation process towards desired results can be seen as quite natural because it usually involves interactions with data which are familiar to the user itself, the same thing cannot be said for the data refinement, in that, data issued by a segmentation phase, which need (for reasons and under hypotheses that will be examined) a refinement phase, are not so familiar to 


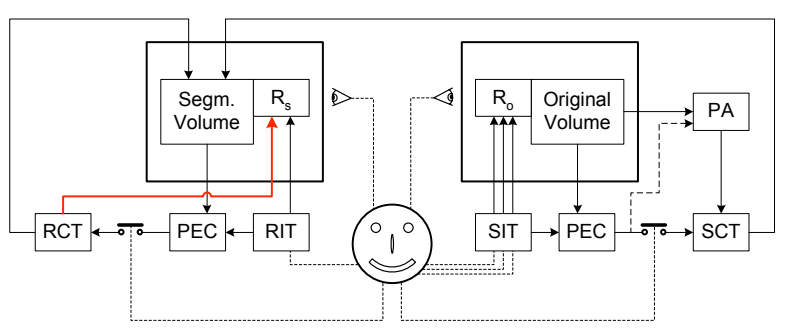

Figure 1. Interactive Segmentation Model.

the user. Thus, a refinement task can quickly became very difficult for the user itself, especially when 3D data (with associated interaction difficulties) are involved.

In this work, we focalize our investigation on the refinement part of a complete interactive segmentation system, by prospecting and implementing what here is called twoway interactive system, where the user guides the process during the segmentation phase (as is of common practice in an interactive system) while, during the refinement phase, instead of being generated by the user, prior information is generated by the computational part of the system. In other word we propose a user-is-aided refinement, and the overall process could be seen as a mixed initiative interactive segmentation system [1].

In Sec. 2 we overview the design principles and a concrete reference system from which we start to introduce the user$i s$-aided refinement described in Sec.3. A meaningful case is presented in Sec.4 to demonstrate the performance and better understand the fields of application of the proposed solution. Conclusions are given in Sec.5.

\section{Two-way interactive segmentation}

\subsection{General paradigm}

The design of an interactive segmentation system (ISS) according to human-centered principles must keep the user into the loop. As already stated, in high generality systems intermediate results should be refinable according to proper (context dependent) visual data interaction loops and the overall human-centered or mixed initiative system activities should guarantee a suitable degree of convergence towards desired results.

In Fig. 1 we present a ISS user-centered paradigm which is suited to represent a wide class of image and volume segmentation problems and solutions. This has been conceived to be a reference for the design and implementation of the proposed system. The simple underlying philosophy is that the user (typically a physician) guide the segmentation and refinement process on the base of a morphological coherence of the structures he want to isolate and that he quickly indicate his target without fully exploring this coherence and by delegating this time burdening task to the segmentation and refinement computational tools (SCT and RCT). In the scheme the user is represented with the smiling face and his actions with dashed lines. One or many segmentation and refinement interaction tools (SIT and RIT) can be thought as elements of suitable segmentation and refinement toolboxes respectively. SIT and RIT should be designed, presented and selected according to the users feeling and the knowledge of their professional culture and working skills. They should be also complete in representing different tasks and robust in providing different ways to communicate the same information. Relevance and adequacy of the interaction tools reside in the possibility to extract from the data interaction itself, a set of quantitative parameters representing what the user would like to communicate (which could be different from one tool to another). These parameters are estimated and calculated into the PEC blocks. These are the physical interface between SIT and RIT and the SCT and RCT respectively, which must be capable to fully exploit such information. A preprocessing analysis (PA) computational block also appears in the scheme. This block is separated from the SCT because of its peculiar role into a segmentation pipeline. SIT and RIT access the rendering engines $\left(R_{o}\right.$ and $\left.R_{s}\right)$ which are responsible of the visualization of the original and segmented data-sets (or a meaningful combination of them) and of the visual feedbacks given to the physician about the carried out actions. The red arrow indicate a particular information path which gives to RCT a direct access to the rendering engine $R_{s}$. This actually invert the flow of information in the sense anticipated in the introduction and will constitute the main focus of the present work. Finally, the circuit closing buttons reminds that the activation of the computational tools is intentional and under the control of the user.

\subsection{Reference system}

An efficient implementation of the above paradigm based on advanced morphological operators, versatile and consistent interaction tools and volume rendering capabilities has been used here. This implementation was initially described in some previous works [2] [6] and recently implemented in an optimized software and experimented in a clinical setting [5]. The segmentation core (SCT) is based on a bounded (according to prior information deriving from user interaction) and optimized 3D watershed segmentation; the preprocessing (PA) consists in a volume filteringby-analysis performed by $3 \mathrm{D}$ connected operators and it is oriented to minimize over-segmentation problems that may arise. Basic refinement RIT and RCT tools, such as local cuts and local erosions, have been also implemented which allow to handle simpler refinement cases, while more intel- 
ligent cuts are addressed, for the first time, in this work.

\section{User-is-aided morphological refining}

As stated above, once a segmented volume has been generated there could be the need of a step of refinement. A typical situation to face is when the desired structures have been correctly segmented but they result physically linked to "undesired" voxel aggregations which make subsequent steps, such as visualization or quantification, unserviceable. In a common case, undesired structures are linked because they are actually connected to the target Structure of Interest $(S o I)$ by means of anatomical structures (e.g. nerves) that may have similar pixel values. In other cases the limits in terms of noise, resolution, tissue discrimination power of the imaging system determine undesired, unpredictable, and potentially sparse, points of contact on the voxel map (lack of separation in terms of gradient information) between structures of similar pixel values which instead, considering the actual anatomy, should appear separated (e.g. by membrane). In these and other similar cases the segmentation did its best in the sense that the user correctly guided the computational tools to use all disposable a-priori and data-derived information (gradient, pixel range, connectivity, markers,...) and their recognition capability in order to isolate the desired SoI. However, depending on applications and due to its inherent unpredictability, information may not be complete in order to let the segmentation produce the desired result. In some newsworthy approaches (e.g. [3]) a higher degree of information is extracted by general anatomical knowledge (atlas-based techniques). However, it is difficult to completely map the knowledge about anatomical variability and pathological situations. In the proposed approach, we try to identify and exploit another form of general anatomical knowledge which characterize the concrete situation which is determined after our interactive segmentation.

From the above observations, we formulate a narrowing (or stricture) hypothesis about the kind of connections among SoI's and undesired regions. Within this framework a refinement step is conceptually akin a surgical dissection and our objective becomes aiding the user to accomplish such a delicate task. We collect a supporting experimental evidence of such hypotheses in different applications and anatomical districts: more extended connections are usually symptom of belonging to the same anatomical structure. Moreover, in possible cases of extended connections between different structures, some problems hardly tractable within a connection-based segmentation framework would arise. Such exceptions are also out of our scope because extended connections are visually noticeable and their slicing can be supported with simpler tools. Another salient characteristic of the proposed solution is that it allows to main-

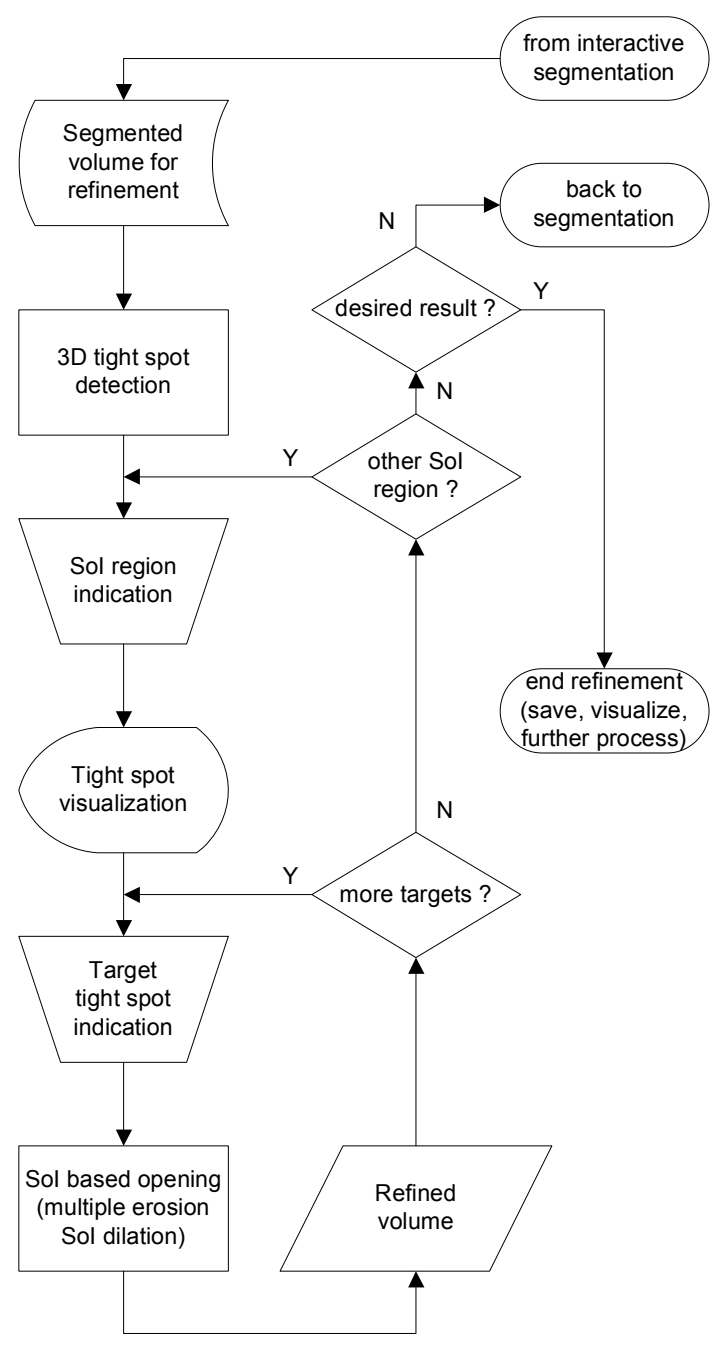

Figure 2. Refinement procedure.

tain a low computational and methodological complexity. Narrowing detection is quite simple to implement and the related underlying hypothesis allow the user to work with suitable tools towards a rapid convergence of the result.

\subsection{General scheme}

Fig. 2 gives a global view of the cooperative refinement procedure which has been designed and implemented according to the two-way interaction paradigm. The following description summarize the process by enumerating its phases.

1. Given a segmented volume $S$ issued from an interactive segmentation phase, a global and automatic narrowing detection is performed on a binarized version 
$S_{b}$ of $S$, which generates a global narrowing map $N_{\text {map }}$.

2. The user indicate on $S$ a region of the $S o I$ he wants to refine by placing a marker seed $M_{1}$.

3. A region growing is performed from $M_{1}$ to $S_{b}$ boundaries and $N_{\text {map }}$ elements: under the narrowing hypotheses a region $R\left(M_{1}\right) \subseteq S o I$ is detected and a new visual representation $V\left(S, M_{1}\right)$ of $S$ is presented to the user which highlight the subset $N\left(M_{1}\right) \subseteq N_{\text {map }}$.

4. The user graphically interact with $V\left(S, M_{1}\right)$ by selecting a set of $k_{1}$ narrowing suspected to be connected to undesired structures.

5. A modified and localized morphological opening is performed: local erosions are performed around the $k_{1}$ targets and a $S_{b}$ re-growing followed by corresponding local dilation complete the operation. This actually generate a refined volume $S_{r}$.

6. The process ends if $S_{r}$ satisfies the user, otherwise

(a) The process can resume from step 4 for other sets of $k_{i}$ narrowings, $i>1$.

(b) The process can resume from step 2 for other $S o I$ regions $R\left(M_{j}\right)$ from related markers $M_{j}, j>1$.

(c) Another segmentation phase required.

More detail about single phases is reported in the following subsections.

The implemented solution is one of the possible way to realize the idea of cooperative (two-way) user-aided with useris-aided refinement, and that it well fits into the proposed segmentation paradigm. A criterion followed here was to let the user take actions on suspected narrowings connecting undesired regions without burdening the computational part in exhaustively exploring such hypotheses. In fact, except for simpler cases, such exploration would become very complex, in that, it is worth noting that there is no algorithmic knowledge about the actual $S o I$ during the refinement phase but only a $R\left(M_{i}\right) \subseteq S o I$ can be estimated. Moreover, the same $S o I$ can be more or less populated of inner narrowings. Therefore even if markers are placed near possible connections to undesired regions, it is not evident how distinguishing what is inside from what is outside the SoI.

\subsection{D narrowing detection}

Narrowing are classified according to a connectivity and dimension based reasoning. For each voxel $v=$ $v(x, y, z)$ of the binarized $S_{b}$ volume a 18-voxels neighborhood $\eta_{18}(v)$ is examined as a result of the application of the 3 orthogonal cutting planes $p \in\{X, Y, Z\}$ passing for

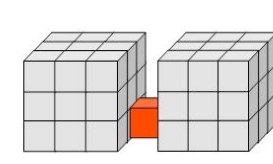

1 voxel

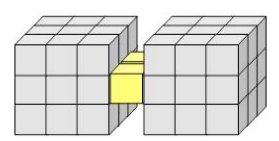

2 voxels

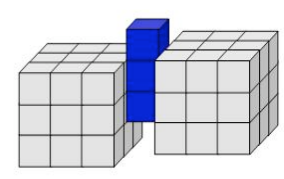

3 voxels

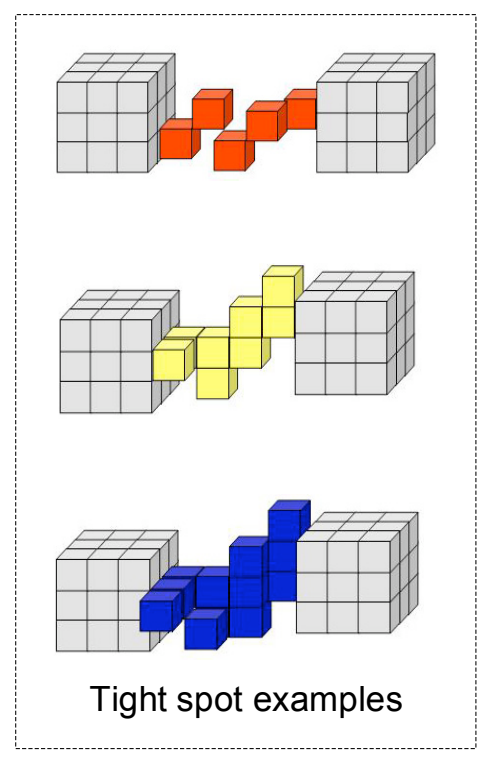

\section{Figure 3. Narrowings: basic kinds (left), same kind composites (right).}

$v$. For each plane all connected components $C(p, v)$ which include $v$ and which dimension is $d \leq Q$ are detected. For each $c \in C(p, v)$ the same $c$ is classified to be (or to belong to) a narrowing if: considering the two planes parallel to $p$ and adjacent to $v$, they both contains connected components which are also connected to $c$ and have at least the same dimension of $c$. In the presented implementation $\mathrm{Q}$ has been fixed to 3. Simple kinds of narrowing are depicted in Fig.3.

\subsection{Local data interaction and processing}

In the following some more details on the interaction and processing during the user-is-aided refinement, described in Sec.3.1 are given. Fig. 4 shows a pictorial representation of the salient phases of the process to which the discussion will refer using parenthesis.

After the global narrowing detection and classification a $N_{\text {map }}=\left\{n_{i}\right\}$ is created (Fig.4(a) where $i=1, \ldots, 4$; note that the segmented volume $S$ contains one $S o I$ and two undesired connected regions $A$ and $B$ ). The user places a marker $\left(M_{1}\right)$ in a region of the $S o I$ in order to try to remove an undesired region (region A). A 3D region growing from that marker is then performed with a contour preserving cubic dilation element. Growing finishes upon reaching one of the following conditions: 1) region boundaries, 2) narrowings. Only the narrowings touched by the growing are then visualized using different colors for different narrowing dimensions: this is to avoid confusing the user with visual 

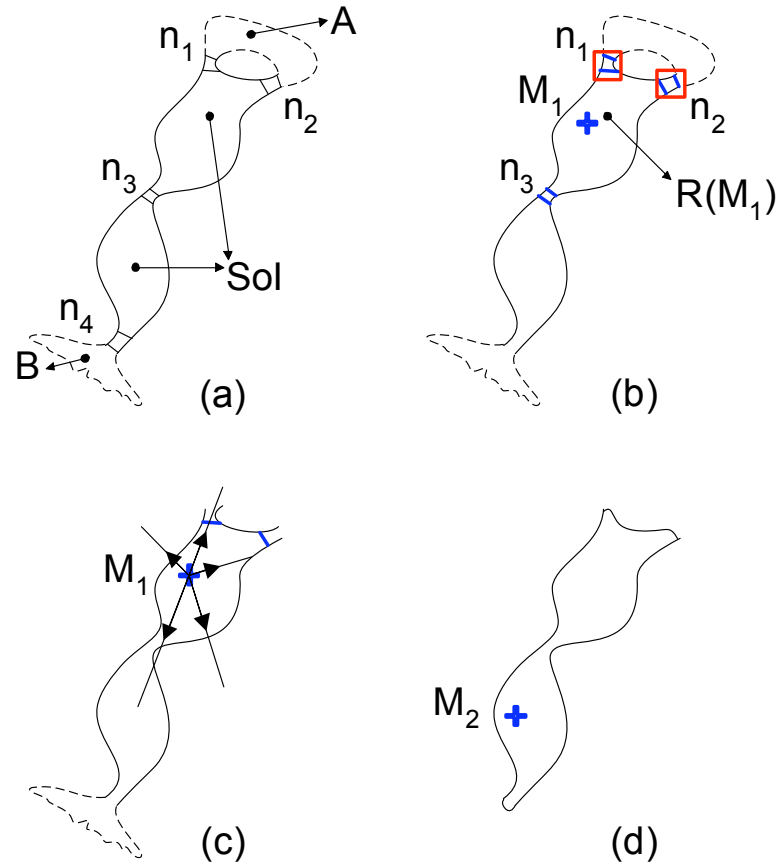

(d)

Figure 4. User-is-aided refinement phases.

information about narrowings not in the proximity of $M_{1}$ (in Fig.4(b) the user sees three $n_{i}$ with $i=1, \ldots, 3$, two of them at the frontier with region A while one internal to the $S o I)$. Looking at $V\left(S, M_{1}\right)$ the user has the implicit possibility to verify that, with respect to which can be joined by the marker $M_{1}$, the undesired region is actually connected only by narrowings. In fact, due to the stop criterion 2), no narrowing should be evidenced on the undesired regions. Subsequently, thanks to the suggestions deriving from $V\left(S, M_{1}\right)$, the user indicate $k_{1}$ regions where narrowings should be truncated (red rectangles in Fig.4(b), $k_{1}=2$ ). In these regions, set to be 3D blocks around the point of selection, a local morphological erosion is performed with a 18-connected 3D structuring element. This actually cut off the narrowings populating these $k_{1}$ regions. Then, a region re-growing follows from the marker $M_{1}$ (Fig.4(c)) with the aim to exclude undesired regions from the subsequent steps. In order to compensate from erosion, dilation is performed locally on the same $k_{1}$ regions, thus realizing a $S o I$-based (or marker-based) morphological opening. In case of residual undesired regions to eliminate (region B in Fig.4(a)) more narrowings can be cut off or another marker can be placed (marker $M_{2}$ Fig.4(d)) and the process can resume as described in Sec.3.1 until satisfaction (Fig.4(d)).

All described functionalities have been implemented in a $\mathrm{C}++$ software prototype which let the user to freely navigate and interact on the three orthogonal slicing directions.

\section{Results and discussion}

In this section we report a meaningful example representing situations the complete system can handle, in particular evidencing the role and the potential of the refinement part. Salient phases are supported by the representation of Fig.5. The original data-set is a $256 \times 256 \times 141$ voxels T1weighted MRI brain scan (rendered in the up-left corner of Fig.5) and the problem is to accurately segment the gray matter. The refinement starts on the segmented volume produced by the interactive segmentation system (Sec.2.2). After the segmentation the gray matter is correctly represented in the segmented data but a certain amount of undesired structures is also included which let the data unserviceable neither for visual nor for quantitative usages. In addition it is very difficult for the user understanding where such undesired voxels are connected and it is also difficult to interact with such kind of unusual 3D data-set. By navigating in 2D slices (according to different slicing directions) the user suspect connections near the region where he place a first marker $M_{1}$. Narrowings are then evidenced and thanks to the related augmented view $V\left(S, M_{1}\right)$ the user is able to manoeuver his virtual scalpel pinpointing the structure to eliminate (in this case only 5 selections was enough). With the indication of the presence of narrowings the user can also better perceive to operate on 3D data even continuing to interact with 2D views. In conclusion of this phase the user decide to suture by letting a new volume to be created in consequence of the described region based opening. This is represented in Fig.5(b): a great reduction of unwanted voxels has been reached but another refinement phase become necessary. User attention now focuses on suspected connections around eyes and optical nerves regions. A second marker $M_{2}$ is placed and few visually assisted dissections (erosions) performed. This second phase finishes (enabling a new local opening) and the desired result produced as shown in Fig.5(c). The system has been tested with success on several other similar and different cases and, despite the refinement part have not yet been optimized in terms of computational cost, no significant burden was reported in terms of computation time, during the refinement interaction. While in some simpler cases the presented two-way interactive segmentation could be seen as a comfort option, in other ones, such as the presented one, it turns out to be a crucial resource.

\section{Conclusions}

In this paper we presented an user-centered refinement technique of segmented volumes based on a general hypothesis of narrowing connections between SoI's and undesired regions. Such approach is particularly indicated for complex segmentation tasks where both accuracy and generality 

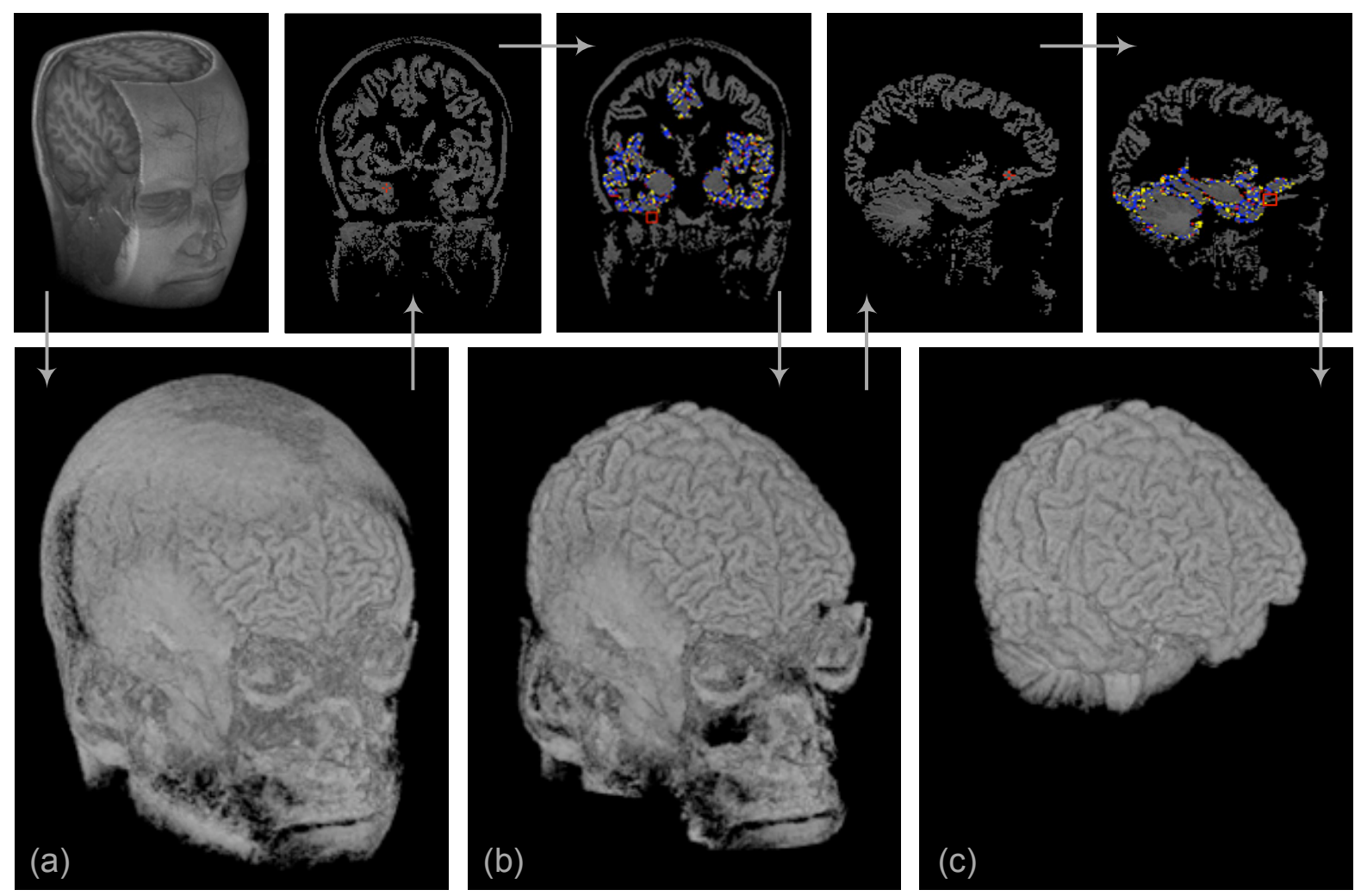

Figure 5. Advanced refinement example: gray matter separation.

of the method are to be preserved. The presented method can be figured as a mixed initiative interactive system. In fact, interactive and computational parts as well as segmentation and refinement steps have been designed to share the same task abstractions and take cooperative actions.

The proposed solution can be considered as a valid alternative to highly specialized segmentation systems and allows to handle a variety of clinical segmentation tasks both for normal and pathological situations. The technical content of this work has been intentionally and mainly oriented to the methodological precision. On this base, a quite simple processing in the refinement subsystem already demonstrated promising results. More evolved processing about narrowing characterization and classification should be addressed in future works in order to improve the quality and usability of the information given to the user or to introduce some useful refinement automatisms.

\section{Acknowledgments}

We would like to thanks our former student Manuel Vega which worked on software implementations and the Neuroradiolgy Unit of the Civil Hospital of Brescia, headed by
Prof. Roberto Gasparotti, for fruitful discussion and collaboration during the experimental phases.

\section{References}

[1] Mixed-initiative interaction. IEEE Intelligent Systems, 14(5):14-23, Sept/Oct 1999. M.A. Hearst (ed.).

[2] S. Benini, E. Boniotti, R. Leonardi, and A. Signoroni. Interactive segmentation of biomedical images and volumes using connected operators. In ICIP 2000, volume 3, page 452455, 2000.

[3] V. Grau, A. Mewes, M. Alcaiz, R. Kikinis, and S. Warfield. Improved watershed transform for medical image segmentation using prior information. IEEE Trans. Medical Imag., 23(4):447-458, April 2004.

[4] S. D. Olabarriaga and A. W. M. Smeulders. Interaction in the segmentation of medical images: A survey. Med Imag Anal, 5:127-142, 2001.

[5] A. Signoroni, R. Grazioli, F. Carletti, R. Leonardi, and R. Gasparotti. An accurate interactive 3D segmentation system with applications to MR brain quantification. In Information Processing in Medical Imaging, IPMI 2007, 2007. paper under review.

[6] A. Signoroni and R. Leonardi. Volume simplification and segmentation by 3D connected operators. In EUSIPCO 2002, volume 3, page 489492, 2002. 